\title{
Gilteritinib, a FLT3/AXL inhibitor, shows antileukemic activity in mouse models of FLT3 mutated acute myeloid leukemia
}

\author{
Masamichi Mori $^{1}$ - Naoki Kaneko ${ }^{1} \cdot$ Yoko Ueno $^{1} \cdot$ Masaki Yamada $^{2} \cdot$ Ruriko Tanaka $^{1}$. \\ Rika Saito $^{1} \cdot$ Itsuro Shimada $^{1} \cdot$ Kenichi Mori $^{1} \cdot$ Sadao Kuromitsu $^{1}$
}

Received: 8 March 2017 / Accepted: 21 April 2017 / Published online: 17 May 2017

(C) The Author(s) 2017. This article is an open access publication

\begin{abstract}
Summary Advances in the understanding of the molecular basis for acute myeloid leukemia (AML) have generated new potential targets for treatment. Fms-like tyrosine kinase 3 (FLT3) is one of the most frequently mutated genes in AML and mutations in this gene are associated with poor overall survival. AXL plays a role in the activation of FLT3 and has been implicated in the pathogenesis of AML. The studies reported here evaluated the ability of a novel FLT3/AXL inhibitor, gilteritinib, to block mutated FLT3 in cellular and animal models of AML. Initial kinase studies showed that gilteritinib, a type I tyrosine kinase inhibitor, was highly selective for both FLT3 and AXL while having weak activity against c-KIT. Gilteritinib demonstrated potent inhibitory activity against the internal tandem duplication (FLT3-ITD) and FLT3-D835Y point mutations in cellular assays using MV411 and MOLM-13 cells as well as Ba/F3 cells expressing mutated FLT3. Gilteritinib also inhibited FLT3-F691 mutations, although to a lesser degree, in these assays. Furthermore, gilteritinib decreased the phosphorylation levels of FLT3 and its downstream targets in both cellular and animal models. In vivo, gilteritinib was distributed at high levels in xenografted tumors after oral administration. The decreased
\end{abstract}

Electronic supplementary material The online version of this article (doi:10.1007/s10637-017-0470-z) contains supplementary material, which is available to authorized users.

Masamichi Mori

masamichi.mori@astellas.com

1 Research Program Management Office, Drug Discovery Research, Astellas Pharma Inc., 21 Miyukigaoka, Tsukuba-shi, Ibaraki 305-8585, Japan

2 Pharmacology Research Division, Astellas Research Technologies Co., Ltd, 21 Miyukigaoka, Tsukuba-shi, Ibaraki, Japan
FLT3 activity and high intratumor distribution of gilteritinib translated to tumor regression and improved survival in xenograft and intra-bone marrow transplantation models of FLT3driven AML. No overt toxicity was seen in mouse models treated with gilteritinib. These results indicate that gilteritinib may be an important next-generation FLT3 inhibitor for use in the treatment of FLT3 mutation-positive AML.

Keywords Acute myeloid leukemia $\cdot$ Fms-liketyrosinekinase $3 \cdot$ Mutation · Protein kinase inhibitors - Xenograft antitumor assays

\section{Introduction}

Acute myeloid leukemia (AML) is a rapidly progressing hematologic cancer characterized by loss of normal differentiation and uncontrolled proliferation of hematopoietic progenitor cells in the bone marrow [1]. This dysregulation of blood cell production leads to anemia, neutropenia, and thrombocytopenia [1]. First-line treatment for patients (aged $\leq 60$ years) with AML often begins with a cytarabine- and anthracycline-based chemotherapy regimen through remission induction and postremission phases. While the complete remission rate for patients who undergo this line of treatment is over $70 \%$ [1], the 5-year survival rate is still only 50\% [2]. Age and leukemia cytogenetics affect patient response rate [2-5], and therapeutic outcomes are worse for patients who are not eligible for the intensive treatment or who are relapsed/refractory to front-line therapy $[6,7]$. A number of advances have been made in understanding the genetics behind AML $[2,3]$ that may lead to new opportunities for the development of targeted therapies.

Fms-like tyrosine kinase 3 (FLT3) is a member of the class III receptor tyrosine kinase (TK) family that is normally expressed on the surface of hematopoietic progenitor cells 
[8]. The FLT3 receptor plays an important role in proliferation, survival, and differentiation of multipotent stem cells [8], and mutations of FLT3 are the most common molecular alteration in AML. Internal tandem duplications (ITDs) at the juxtamembrane domain within FLT3 are present in 25-30\% of newly diagnosed AML cases [9-12], representing the most frequent FLT3-activating mutation. Activating point mutations within the FLT3 TK domain (TKD) are also observed in patients with AML, but with less frequency than ITD mutations $[11,13]$. These activating mutations are oncogenic and are associated with poor prognosis, higher relapse rate, more rapid time to relapse, reduced disease-free survival, and reduced overall survival, although the prognostic impact for FLT3TKD mutations is controversial $[11,12,14,15]$. Overall, these findings suggest that FLT3 is a potential therapeutic target for patients with AML who are harboring a FLT3 mutation.

Similar to FLT3, AXL (a member of the TAM family of receptor TKs) has transforming properties and has been identified as a potential therapeutic target for AML $[16,17]$. Preclinical studies showed that inhibition of AXL blocks proliferation of FLT3 mutant and FLT3 wild-type AML cells and also suppresses the leukemic burden of FLT3-ITD ${ }^{+}$AML in both a subcutaneous xenograft model and a leukemia engraftment model [16-18]. Furthermore, activated AXL may be required for resistance to FLT3 inhibitors [19]. Therefore, a new chemical entity that targets both FLT3 and AXL may provide a novel treatment option for AML.

Gilteritinib is a small-molecule FLT3/AXL inhibitor with a structure based on a pyrazine carboxamide scaffold. The research described here aimed at characterizing the activity of gilteritinib against FLT3-driven AML in preclinical models. The inhibitory effects of gilteritinib against FLT3 were assessed in cell-free systems and cellular assays, and its antitumor activity and survival benefits were evaluated in FLT3driven tumors in mice.

\section{Materials and methods}

\section{Compounds, cell lines, and antibodies}

Small molecule TK inhibitors (TKI) gilteritinib hemifumarate (gilteritinib; ASP2215) and quizartinib were synthesized by Astellas Pharma Inc. (Tokyo, Japan). Both gilteritinib and quizartinib were dissolved in DMSO or suspended in $0.5 \%$ methylcellulose for in vitro or in vivo experiments. MV4-11 cells were purchased from the American Type Culture Collection (ATCC, Manassas, VA); MOLM-13 cells were purchased from the German Collection of Microorganisms and Cell Cultures (Braunschweig, Germany). MV4-11-luc cells, which exogenously express firefly luciferase, were prepared at Astellas Pharma Inc. (a detailed protocol for the generation of the constructs is found in the online resource).
MV4-11-AXL cells, which exogenously express AXL, were also prepared at Astellas Pharma Inc. $\mathrm{Ba} / \mathrm{F} 3$ cells expressing FLT3-ITD, FLT3-D835Y, FLT3-ITD-D835Y, FLT3-ITDF691 L, or FLT3-ITD-F691I mutations (FLT3-ITD Ba/F3, FLT3-D835Y_Ba/F3, FLT3-ITD-D835Y_Ba/F3, FLT3-ITDF691L Ba/F3, FLT3-ITD-F691I Ba/F3) were established by Astellas Pharma Inc. The following antibodies were used for immunoblotting: FLT3 (Abcam, Cambridge, MA); phosphorylated pan-tyrosine (Merck Millipore, Billerica, MA); p44/42 MAPK (ERK1/2), phospho-p44/42 MAPK (ERK1/2) (Thr202/Tyr204) (197G2) (E10), AKT, phospho-AKT (Ser473) (193H12), and AXL (C44G1) (Cell Signaling Technology, Danvers, MA); STAT5 and phospho-STAT5 (Y694) (BD Biosciences, Franklin Lakes, NJ); phosphoAXL (Y779) (MAB6965, R\&D Systems, Minneapolis, $\mathrm{MN}$ ); and $\beta$-actin (Sigma-Aldrich, St. Louis, MO).

\section{Kinase inhibitory assays}

The kinase inhibitory activity of gilteritinib was tested against a panel of 78 TKs (Table S1) using ATP concentrations that were approximately equal to the $\mathrm{K}_{\mathrm{m}}$ value for each kinase in a TK-ELISA or off-chip mobility shift assay (MSA) at Carna Biosciences, Inc. (Kobe, Japan). These assays were conducted according to the manufacturer's instructions. Initially, two concentrations of gilteritinib ( $1 \mathrm{nM}$ and $5 \mathrm{nM}$ ) were tested to assess each compound's inhibitory effect on TK activity. Further studies were then conducted using a dose range of gilteritinib to determine $\mathrm{IC}_{50}$ values for kinases in which activity was inhibited by $>50 \%$ with $1 \mathrm{nM}$ gilteritinib as well as for c-KIT. TK-ELISA and MSA assays were used to conduct $\mathrm{IC}_{50}$ studies for FLT3, LTK, AXL, and c-KIT; the HTRF® KinEASETM-TK assay (Sceti Medical Labo, Tokyo, Japan) was performed according to the manufacturer's protocol to assess the $\mathrm{IC}_{50}$ value of echinoderm microtubule-associated protein-like 4-ALK (EML4-ALK; three individual experiments for each kinase).

\section{Cell viability}

The effect of gilteritinib on MV4-11 and MOLM-13 cells was assessed using the CellTiter-Glo® Luminescent Cell Viability Assay (Promega, Madison, WI). Subsequent studies were conducted to examine the effect of gilteritinib and quizartinib on $\mathrm{Ba} / \mathrm{F} 3$ cells expressing either FLT3-ITD, FLT3-D835Y, FLT3-ITD-D835Y, FLT3-ITD-F691 L, or FLT3-ITD-F691I. A detailed protocol is provided in the online resource.

\section{Immunoprecipitation and immunoblotting}

FLT3 immunoprecipitation and immunoblotting for all targets were performed following standard protocols. Detailed methods are provided in the online resource. 


\section{Animal models for in vivo studies}

All animal experimental procedures were approved by the Institutional Animal Care and Use Committee of Astellas Pharma Inc., and the Tsukuba Research Center of Astellas Pharma Inc. was awarded accreditation status by AAALAC International. Mice were maintained on standard diet and water throughout experimental procedures.

\section{In vivo mouse xenograft model}

Details on the cell engraftment for the mouse xenograft model and the dosing protocols are provided in the online resource.

\section{Pharmacokinetics}

Nude mice subcutaneously xenografted with MV4-11 cells received gilteritinib suspended in a $0.5 \%$ methylcellulose solution as an oral single dose. Blood samples were collected at protocol-specified time points from the inferior vena cava using a syringe with EDTA-2Na, and plasma samples were prepared by centrifugation ( $n=3$ per group; five plasma samples showed abnormal results and were not used for data analysis). Tumor samples were also collected from each mouse and tumor weight was measured. The plasma and tumor concentrations of gilteritinib were measured using highperformance liquid chromatography-tandem mass spectrometry at Nemoto Science (Ibaraki, Japan). Standard pharmacokinetic parameters $\left(\mathrm{C}_{\max }, \mathrm{T}_{\max }\right.$, and $\left.\mathrm{AUC}_{\mathrm{t}}\right)$ were calculated from the mean concentrations of gilteritinib using WinNonlin V6.1 (Certara, Princeton, NJ).

\section{In vivo xenograft mouse studies for gilteritinib kinase inhibitory activity}

The in vivo gilteritinib kinase inhibitory activity was assessed using tumor protein lysates from the xenograft mouse model in an ELISA for phosphorylated-FLT3 and total FLT3. Gilteritinib inhibitory activity was also confirmed by analyzing phosphorylated-STAT5 levels. Detailed protocols can be found in the online resource.

\section{In vivo xenograft studies for gilteritinib antitumor activity}

Once tumor growth had been confirmed, mice xenografted with MV4-11 cells were monitored for 28 days; mice xenografted with $\mathrm{Ba} / \mathrm{F} 3$ cells expressing FLT3 mutations were monitored for 7 days. Tumor diameter was measured using a caliper, and tumor volume was determined by calculating the volume of an ellipsoid using the formula: length $\times$ width $^{2} \times 0.5$. Body weight was measured using a standard balance. Data are expressed as mean \pm SEM from $n=6$ mice/group (MV4-11) and $n=5$ mice/group $(\mathrm{Ba} / \mathrm{F} 3)$.

\section{Intra-bone marrow transplantation model}

In the intra-bone marrow transplantation (IBMT) model, MV4-11-luc cells $\left(1 \times 10^{6}\right.$ cells/mouse $)$ were injected into the bone marrow of the left tibia of female NOD-SCID mice (day 0 ) following the protocol detailed by Lee et al. [20]. Tumor growth was monitored by bioluminescent imaging of the whole body with an IVIS Spectrum (PerkinElmer, Waltham, MA). After confirming tumor cell engraftment at day 14 , mice were orally administered with once-daily vehicle control or gilteritinib at $30 \mathrm{mg} / \mathrm{kg}$ from day 15 to day 70 $(n=10)$. Tumor growth was monitored once a week during the dosing period and then every other week until day 100 . Survival was also monitored daily until day 168 .

\section{Computational modeling}

Docking simulation of gilteritinib with FLT3 was performed using the docking software GLIDE implemented in Maestro version 9.7 (Schrodinger, LLC, New York, NY). The coordinate of ligand-protein binding for FLT3 was modeled based on the ATP-binding form of cKIT, which had the most similar kinase domain amino acid sequence in the Protein Data Bank (PDB) (PDB ID: 1PKG, chain A) and, therefore, was used as a surrogate for FLT3. The homology modeling was done by the modeling software MOE (Chemical Computing Group Inc., Montreal, Quebec, Canada). Hydrogen atoms were added using the computer program Protonate3D implemented in MOE. The docking mode with the highest docking score was employed. All molecular visualization was produced by MOE.

\section{Statistical analyses}

For the in vivo subcutaneous xenograft mouse model, values are expressed as mean \pm SEM. Tumor volume and body weight on day 28 in the gilteritinib-treated groups were compared with those in the control group using Dunnett's test. For the IBMT model, the bioluminescence value on day 42 from the gilteritinib-treated group was compared with that from the control group using Student's t-test. The median survival times were compared using the log-rank test. $P<0.05$ was considered significant. SAS software (SAS Institute Inc., Cary, NC), Microsoft Excel (Microsoft, Redmond, WA), and GraphPad Prism (GraphPad Software, La Jolla, CA) were used for data processing.

\section{Results}

\section{Gilteritinib inhibitory activity}

Gilteritinib (structure provided in Fig. 1a) inhibited the activity of eight of the 78 tested kinases by over $50 \%$ at 
a<smiles>CCc1nc(NC2CCOCC2)c(Nc2ccc(N3CCC(N4CCN(C)CC4)CC3)c(OC)c2)nc1C(N)=O</smiles>

b

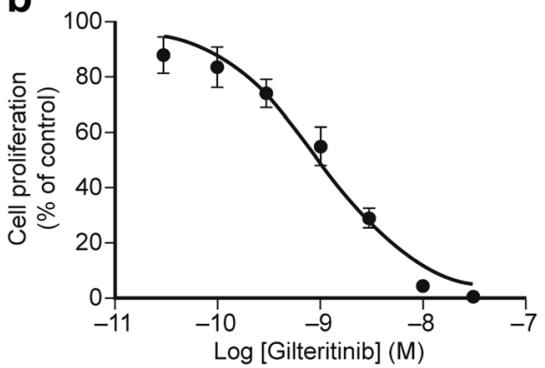

d

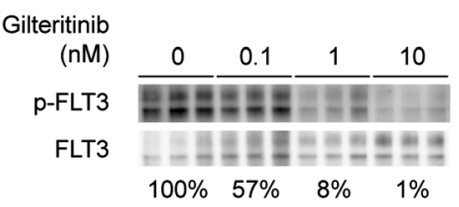

Fig. 1 Gilteritinib inhibits cell growth in AML cells and blocks phosphorylation of FLT3 and its downstream targets. a Structure of gilteritinib (ASP2215). b MV4-11 cells were treated with DMSO or increasing concentrations of gilteritinib for 5 days, and cell viability was measured using CellTiter-Glo. A representative result from three independent experiments is shown. Data are presented as mean \pm SEM (quadruplicate). c MOLM-13 cells were treated in the same manner as MV4-11. A representative result from three independent experiments is

concentrations of either $1 \mathrm{nM}$ (FLT3, LTK, ALK, and AXL) or $5 \mathrm{nM}$ (TRKA, ROS, RET, and MER) (Table S1). The $\mathrm{IC}_{50}$ values were $0.29 \mathrm{nM}$ for FLT3 and $0.73 \mathrm{nM}$ for AXL. Gilteritinib inhibited FLT3 at an $\mathrm{IC}_{50}$ value that was approximately 800 -fold more potent than the concentration required to inhibit c-KIT (230 nM; Table 1).

We next evaluated the antiproliferative activity of gilteritinib against MV4-11 and MOLM-13 cells, which endogenously express FLT3-ITD. After 5 days of treatment, gilteritinib inhibited the growth of MV4-11 and MOLM-13 cells with mean $\mathrm{IC}_{50}$ values of $0.92 \mathrm{nM}(95 \% \mathrm{CI}: 0.23-$ $3.6 \mathrm{nM})$ and $2.9 \mathrm{nM}(95 \% \mathrm{CI}: 1.4-5.8 \mathrm{nM})$, respectively (Fig. 1b, c). Growth suppression of MV4-11 cells was accompanied by inhibition of FLT3 phosphorylation. Relative to vehicle control cells, phosphorylated FLT3 levels were 57\%, $8 \%$, and $1 \%$ after $2 \mathrm{~h}$ of treatment with $0.1 \mathrm{nM}, 1 \mathrm{nM}$, and $10 \mathrm{nM}$ gilteritinib, respectively (Fig. 1d). In addition, doses as

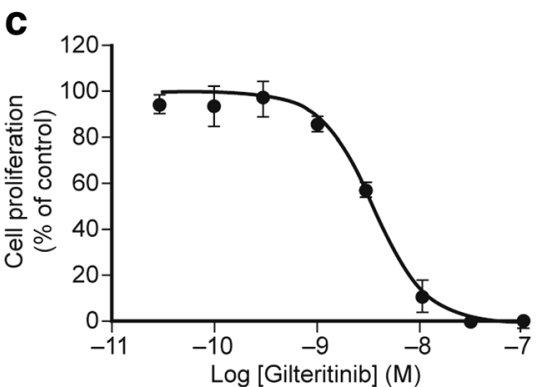

e

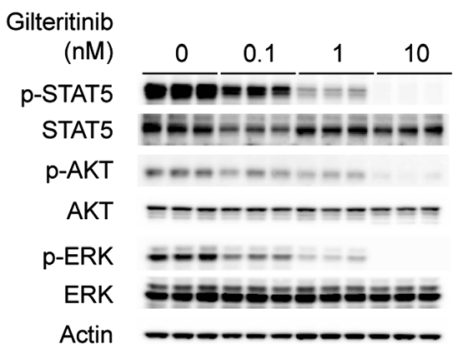

shown. Data are presented as mean \pm SEM (quadruplicate). d Immunoprecipitation and immunoblot for phosphorylated FLT3 and total FLT3 in MV4-11 cells treated with DMSO or increasing concentrations of gilteritinib for $2 \mathrm{~h}$. Blots from one study, which was done in triplicate, are shown. Densitometry values are listed below each treatment group. e Immunoblot for phosphorylated AKT, ERK, and STAT5 in gilteritinib-treated MV4-11 cells. Blots from one study, which was done in triplicate, are shown

low as $0.1 \mathrm{nM}$ or $1 \mathrm{nM}$ resulted in the suppression of phosphorylated ERK, STAT5, and AKT, all of which are downstream targets of FLT3 activation (Fig. 1e, Table S2). To investigate the effects of gilteritinib on AXL inhibition, MV4-

Table 1 Inhibitory activity of gilteritinib against various tyrosine kinases

\begin{tabular}{ll}
\hline Kinase & $\mathrm{IC}_{50}(\mathrm{nM})(95 \% \mathrm{CI})$ \\
\hline FLT3 & $0.29(0.26-0.32)$ \\
LTK & $0.35(0.29-0.43)$ \\
AXL & $0.73(0.51-1.0)$ \\
EML4-ALK & $1.2(0.68-2.0)$ \\
c-KIT & $230(190-280)$ \\
\hline
\end{tabular}

Inhibitory assays for tyrosine kinases were conducted using TK-ELISA, off-chip MSA, or HTRF® KinEASETM-TK. Geometric mean $\mathrm{IC}_{50}$ values were determined from 3 experiments 
11 cells that expressed exogenous AXL were treated with gilteritinib. At concentrations of $1 \mathrm{nM}, 10 \mathrm{nM}$, and $100 \mathrm{nM}$ for $4 \mathrm{~h}$, gilteritinib treatment decreased phosphorylated AXL levels by $38 \%, 29 \%$, and $22 \%$, respectively (Fig. S1).

\section{Pharmacokinetic profile and pharmacodynamic effects of gilteritinib in an MV4-11 xenograft mouse model}

The maximal plasma concentrations of gilteritinib were observed $2 \mathrm{~h}$ after a single oral administration of gilteritinib at $1 \mathrm{mg} / \mathrm{kg}, 6 \mathrm{mg} / \mathrm{kg}$, and $10 \mathrm{mg} / \mathrm{kg}$ to MV4-11 xenografted mice. By contrast, the maximal intratumor concentrations were observed $4 \mathrm{~h}(1 \mathrm{mg} / \mathrm{kg})$ or $8 \mathrm{~h}(6 \mathrm{mg} / \mathrm{kg}$ and $10 \mathrm{mg} / \mathrm{kg})$ after dosing. $\mathrm{C}_{\max }$ and $\mathrm{AUC}_{\mathrm{t}}$ in plasma and tumors increased with increasing doses between $1 \mathrm{mg} / \mathrm{kg}$ and $10 \mathrm{mg} / \mathrm{kg}$. The concentration in tumors was higher than that in plasma at each time point (Fig. 2a-b, Table S3). Gilteritinib dose ranges for cell viability studies are presented in the online resource (Table S4).

Phosphorylated FLT3 decreased by approximately $40 \%$ compared with control phosphorylation levels in tumor samples within $1 \mathrm{~h}$ after single oral administration of $1-10 \mathrm{mg} / \mathrm{kg}$ gilteritinib (Fig. 2c), indicating target inhibition by gilteritinib. Although the decreased phosphorylation state observed in the $1 \mathrm{mg} / \mathrm{kg}$ treatment group had returned to the pre-treatment level by $24 \mathrm{~h}$, the marked decrease in phospho-FLT3 seen with $10 \mathrm{mg} / \mathrm{kg}$ was maintained throughout the $24 \mathrm{~h}$ experimental period. Furthermore, the phosphorylation level of STAT5, a downstream target of FLT3, was almost completely abolished in tumors $4 \mathrm{~h}$ and $8 \mathrm{~h}$ after single administration of gilteritinib at doses of either $6 \mathrm{mg} / \mathrm{kg}$ or $10 \mathrm{mg} / \mathrm{kg}$, respectively. STAT5 phosphorylation levels remained low $24 \mathrm{~h}$ post drug, approaching only $20 \%$ of baseline levels (Fig. 2d). A dose of $3 \mathrm{mg} / \mathrm{kg}$ gilteritinib also decreased phospho-STAT5 to $<20 \%$ of baseline levels at $4 \mathrm{~h}$ and $8 \mathrm{~h}$, but returned to approximately $50 \%$ by $24 \mathrm{~h}$.

The effect on tumor burden of inhibiting FLT3 phosphorylation was assessed following 28 days of once-daily oral administration of gilteritinib. Significant growth inhibition of MV4-11 tumors was observed at $1 \mathrm{mg} / \mathrm{kg} /$ day $(63 \%$ inhibition; $P<0.05)$ and $3 \mathrm{mg} / \mathrm{kg} /$ day ( $80 \%$ inhibition; $P<0.01$ ), and near-complete tumor regression was seen at $6 \mathrm{mg} / \mathrm{kg} /$ day $(93 \% ; P<0.001)$ and $10 \mathrm{mg} / \mathrm{kg} /$ day $(100 \% ; P<0.001)$ (Fig. 2e). Four of the six mice in the $6 \mathrm{mg} / \mathrm{kg} /$ day group experienced complete tumor regression; all six mice in the $10 \mathrm{mg} / \mathrm{kg} /$ day group experienced complete tumor regression. Body weight was not affected by treatment with gilteritinib at any tested dose (Fig. 2f).

\section{Inhibitory activity of gilteritinib against FLT3 containing ITD \pm D835Y or F691 L/I mutations}

Since mutations within the TKD of FLT3 (eg, FLT3D835Y or FLT3-F691) often confer resistance to FLT3 inhibitors that were previously effective against FLT3-ITD [21], the effect of gilteritinib on these resistance mutations was studied. Gilteritinib inhibited the cell growth of $\mathrm{Ba} / \mathrm{F} 3$ cells expressing either FLT3-ITD, FLT3-D835Y, FLT3ITD-D835Y, FLT3-ITD-F691 L, or FLT3-ITD-F691I, with $\mathrm{IC}_{50}$ values of $1.8 \mathrm{nM}(95 \% \mathrm{CI}: 1.0-3.0 \mathrm{nM}), 1.6 \mathrm{nM}(95 \%$ CI: $1.1-2.4 \mathrm{nM}), 2.1 \mathrm{nM}(95 \% \mathrm{CI}: 1.4-3.0 \mathrm{nM}), 22 \mathrm{nM}$ (95\% CI: $15-33 \mathrm{nM})$, and $49 \mathrm{nM}$ (95\% CI: $29-83 \mathrm{nM})$, respectively. The growth of parental $\mathrm{Ba} / \mathrm{F} 3$ cells in the presence of IL-3 was inhibited with an $\mathrm{IC}_{50}$ value of $420 \mathrm{nM}$ (95\% CI: $350-500 \mathrm{nM}$ ), which represents the off-target inhibition of kinases other than FLT3. We confirmed that phosphorylation of FLT3 and its downstream targets STAT5, AKT, and ERK were inhibited by gilteritinib in a dose-dependent manner in $\mathrm{Ba} / \mathrm{F} 3$ cells expressing either FLT3-ITD, FLT3-D835Y, or FLT3-ITDD835Y (Fig. S2). Furthermore, in nude mice xenografted with $\mathrm{Ba} / \mathrm{F} 3$ cells expressing either FLT3-ITD, FLT3D835Y, or FLT3-ITD-D835Y, gilteritinib showed antitumor efficacy at $10 \mathrm{mg} / \mathrm{kg}$ and $30 \mathrm{mg} / \mathrm{kg}$, and induced tumor regression at $30 \mathrm{mg} / \mathrm{kg}$, in all three models (Fig. 3). Following treatment with quizartinib, a second-generation FLT3 inhibitor, the $\mathrm{IC}_{50}$ values for growth inhibition in $\mathrm{Ba} /$ F3 cells expressing FLT3-ITD, FLT3-D835Y, or FLT3ITD-D835Y mutations were $0.46 \mathrm{nM}$ (95\% CI: $0.078-$ $2.8 \mathrm{nM}), 5.7 \mathrm{nM}(95 \% \mathrm{CI}: 1.5-21 \mathrm{nM})$, and $35 \mathrm{nM}(95 \%$ CI: $9.3-130 \mathrm{nM})$, respectively.

\section{Gilteritinib binds to FLT3 at the ATP-binding site}

The finding that gilteritinib inhibited FLT3-D835Y and FLT3ITD-D835Y, both of which harbor mutations in the activation loop essential for binding type 2 inhibitors, suggests that gilteritinib is a type 1 FLT3 inhibitor. To further understand the inhibitory activity of gilteritinib against mutated FLT3 at the structural level, computational modeling was performed for FLT3 with gilteritinib (Fig. 4). The modeling revealed that gilteritinib fits into the active (DFG [Asp-Phe-Gly]-in) conformation of FLT3 at the ATP-binding site, far from the D835 position in the activation loop. The modeling also showed that gilteritinib hydrophobically interacts with FLT3 at the F691 position.

\section{Gilteritinib prolongs survival in mice with IBMT of AML cells}

Treatment with gilteritinib showed a significant reduction in overall bioluminescence, with MV4-11-luc tumor growth dramatically reduced during the first 2 weeks of treatment (Fig. 5a), resulting in bioluminescence levels that were similar to that of background (around $10^{6}$ photons $/ \mathrm{s}$ ) on the penultimate day of treatment (day 70). Furthermore, when control mice showed MV4-11 cell infiltration in several areas of the body, gilteritinib-treated mice had no evidence of cell infiltration (Fig. 5b). Gilteritinib treatment significantly increased survival of MV4-11 xenografted mice and all treated mice 

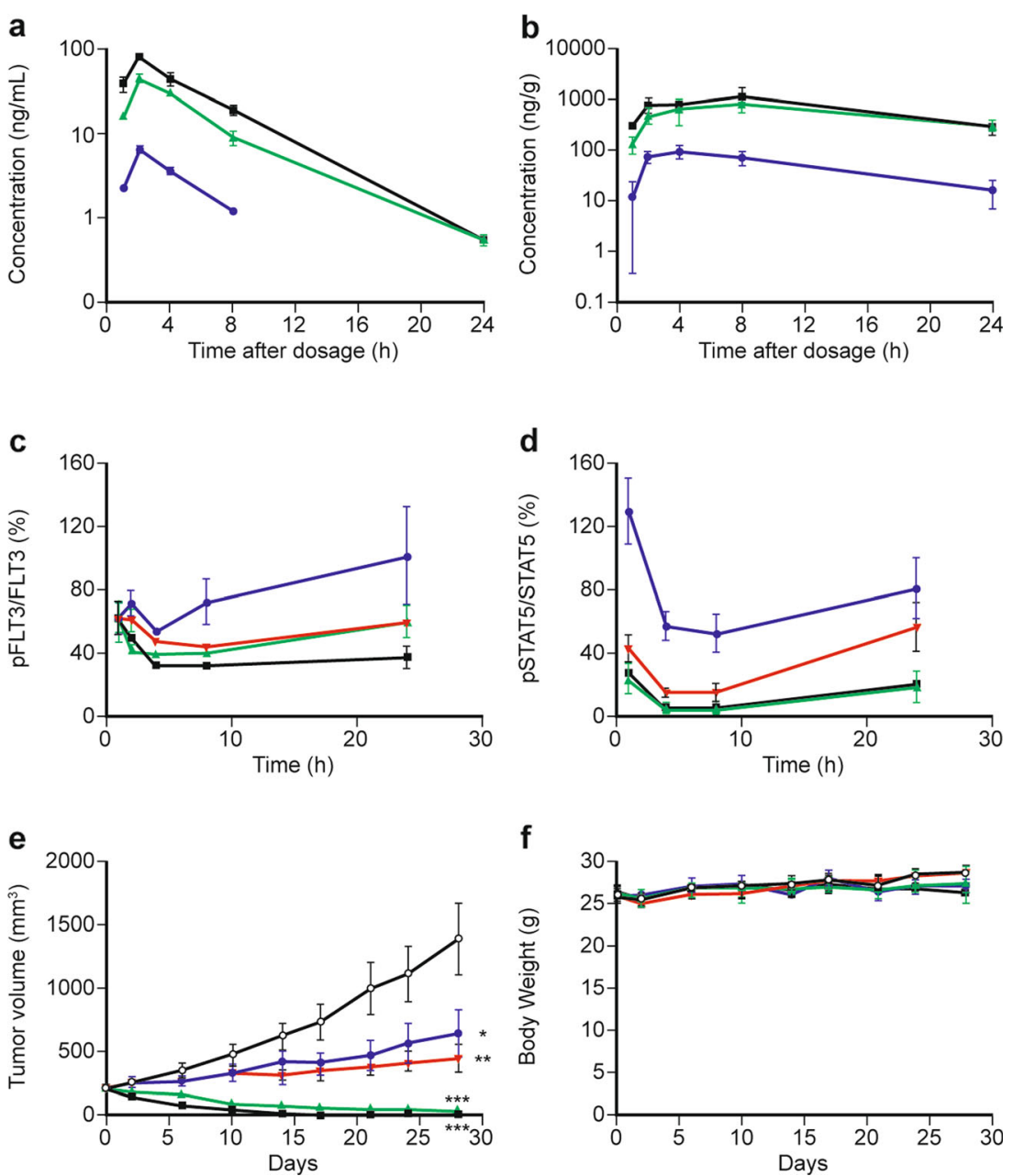

Fig. 2 Antitumor activity of gilteritinib in an MV4-11 xenograft AML mouse model. a Plasma concentrations of gilteritinib were determined by high-performance liquid chromatography-tandem mass spectrometry from nude mice subcutaneously xenografted with MV4-11 cells and treated with a single oral dose of gilteritinib. Data are presented as mean \pm SD for two or three animals. b Tumor concentrations of gilteritinib were determined by high-performance liquid chromatography-tandem mass spectrometry from nude mice subcutaneously xenografted with MV4-11 cells and treated with a single dose of gilteritinib. Data are presented as mean \pm SD for three animals. Symbols represent treatment groups: $1 \mathrm{mg} / \mathrm{kg}(\bullet), 6 \mathrm{mg} / \mathrm{kg}$ (A), $10 \mathrm{mg} / \mathrm{kg}$ (@). c and d Male mice xenografted with MV4-11 cells were orally treated with either vehicle control or increasing

survived to day 168 , whereas vehicle control mice had a median survival time of 61.5 days $(P<0.001)$ (Fig. 5 c).

\section{Discussion}

These data demonstrate that gilteritinib, a FLT3/AXL TKI, shows potent efficacy in preclinical models of FLT3-mutated AML. In vitro kinase assays using gilteritinib revealed strong inhibition of FLT3 and AXL (in the nM range) and an 800-fold weaker inhibition of c-KIT than FLT3. Gilteritinib also inhibited phosphorylation of FLT3 with ITD and/or activation

concentrations of gilteritinib. Protein lysates were collected over a $24 \mathrm{~h}$ time course for assessment of (c) phospho-FLT3 to total FLT3 as determined by ELISA or (d) phospho-STAT5 and total STAT5 as determined by immunoblot. Data are presented as the ratio of phosphorylated protein levels normalized to total protein levels relative to vehicle control-treated lysates. e and $\mathbf{f}$ Male mice xenografted with MV4-11 cells were orally treated with either vehicle control or increasing concentrations of gilteritinib over a 28-day period to examine the effect of drug on (e) tumor volume and (f) body weight. Data presented as mean \pm SEM for $n=6$ mice/group. Symbols represent treatment groups: $1 \mathrm{mg} / \mathrm{kg}(\bullet), 3 \mathrm{mg} / \mathrm{kg}(\boldsymbol{\nabla}), 6 \mathrm{mg} / \mathrm{kg}(\boldsymbol{\Lambda})$, $10 \mathrm{mg} / \mathrm{kg}(\mathbf{\square}) * P<0.05$, $* * P<0.01$, and $* * * P<0.001$ compared with the value of the control group on Day 28 (Dunnett's test)

loop D835Y mutations in cellular assays. The inhibitory activity of gilteritinib against the D835Y mutation is further supported by computational modeling that showed gilteritinib binds to FLT3 within the activation loop far from the D835 site, allowing kinase inhibition to occur in the face of FLT3 mutations at this location. This potent FLT3 inhibition translated to a marked decrease in the cell viability of FLT3 mutationpositive cell lines with inhibition of several downstream targets. In vivo, gilteritinib was distributed at high levels in tumors after single oral administration and showed antitumor activity against FLT3-driven tumors in a mouse xenograft model. This antitumor activity was associated with a durable inhibition 


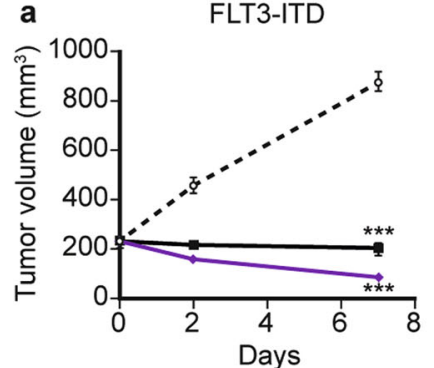

Fig. 3 Gilteritinib induces regression of FLT3 mutant-expressing tumors in a mouse xenograft model. Male nude mice were xenografted with $\mathrm{Ba} /$ F3 cells expressing FLT3-ITD, FLT3-D835Y, or FLT3-ITD-D835Y. Following confirmed tumor growth, mice were treated with either $10 \mathrm{mg} / \mathrm{kg}$ or $30 \mathrm{mg} / \mathrm{kg}$ once-daily oral gilteritinib for up to 7 days. Tumor volume was assessed at specified time points in (a) FLT3-ITD
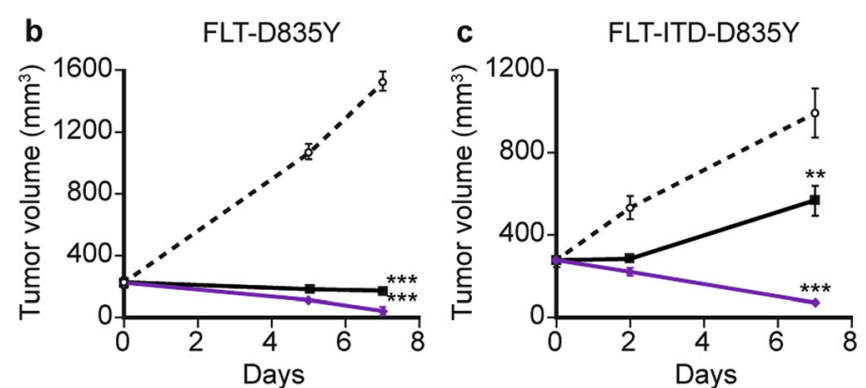

xenografted mice, b FLT3-D835Y xenografted mice, and (c) FLT3ITD-D835Y xenografted mice. Data presented are mean \pm SEM for $n=5$ mice/group. Symbols represent treatment groups: control (०), $10 \mathrm{mg} / \mathrm{kg}(\mathbf{-})$, and $30 \mathrm{mg} / \mathrm{kg}(\diamond) . * * P<0.01$ and $* * * P<0.001$ compared with the value of the control group on Day 7 (Dunnett's test)

of phospho-FLT3 and phospho-STAT5, demonstrating the in vivo inhibitory effect of gilteritinib. Furthermore, treatment with gilteritinib decreased the leukemic burden and prolonged survival in a mouse IBMT model. Taken together, these data suggest that gilteritinib blocks FLT3 phosphorylation, impairs downstream signal transduction, and consequently inhibits AML cell proliferation in both in vitro and in vivo models.

These preclinical findings regarding the activity of gilteritinib suggest it may be a potential therapeutic treatment option for patients with FLT3-mutated AML. Although several TKIs have been investigated as potential therapies for AML, none have yet to be approved for clinical use [2, 9]. First-generation FLT3 inhibitors, such as sunitinib and sorafenib, are pan-kinase

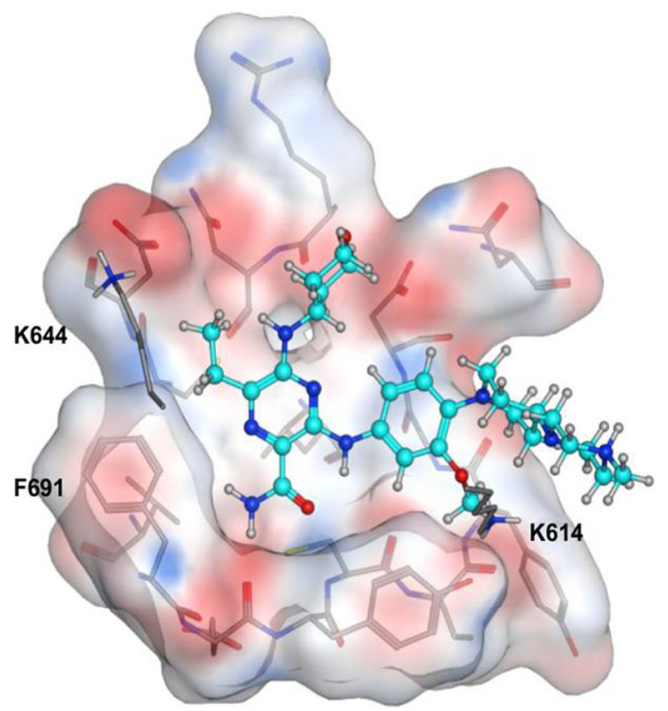

Fig. 4 Computational modeling of gilteritinib binding to wild-type FLT3. Gilteritinib is shown as a ball-and-stick model. All of atoms are colored by the type of element (white: hydrogen; cyan and gray: carbon; blue: nitrogen; red: oxygen). The protein surface is colored by electrostatic potential (blue: positive; red: negative; white: neutral). For clarity, the hydrogen atoms of the protein are omitted with the exception of the polar hydrogen atoms of the side-chains of K614 and K644; similarly, the protein surface in front of the gatekeeper residue F691 is hidden inhibitors that non-selectively target FLT3; this non-specificity often leads to off-target kinase inhibition and severe toxicity in patients [2]. Next-generation inhibitors, such as gilteritinib, crenolanib, and quizartinib, more selectively target FLT3, suggesting less off-target effects and potentially decreased toxicity in patients. The data presented herein suggest that gilteritinib has a potent inhibitory effect on FLT3 with minimal impact on cKIT, demonstrating the specificity of the compound. Studies have shown that mice deficient in both flt 3 and c-kit show a large overall decrease in hematopoietic cell number, whereas mice deficient in only $f t 3$ have normal mature hematopoietic populations with specific deficiencies in primitive B lymphoid progenitors [22]. Thus, the targeted FLT3 inhibition and markedly weaker c-KIT inhibition by gilteritinib suggests a lower clinical risk of myelosuppression than often occurs with other TKIs [23].

When comparing the next-generation FLT3 inhibitors in development, all three compounds exhibit similar activity against FLT3-ITD in vitro, inhibiting the growth of MV4-11 cells at low $\mathrm{nM}$ concentrations (gilteritinib: $0.92 \mathrm{nM}$, crenolanib: $1.3 \mathrm{nM}$, quizartinib: $0.56 \mathrm{nM}$ ) [24, 25]. However, gilteritinib also showed potent inhibition of FLT3-D835Y and FLT3-ITD/ D835Y mutations whereas quizartinib was ineffective at blocking these mutations [26]. Furthermore, we demonstrated that quizartinib had weaker activity against FLT3-D835Y and FLT3-ITD/D835Y mutations compared with FLT3-ITD mutations. Thus, in contrast to quizartinib, gilteritinib has the advantage of effectively blocking FLT3-ITD/D835Y and FLT3D835Y. These preclinical data suggest that gilteritinib may provide improved clinical efficacy even in the presence of the D835Y, a mutation that has been clinically shown to confer resistance to FLT3 inhibitor treatment [27]. In addition, in cellular assays, gilteritinib showed inhibitory activity against F691 mutations, a mutation also detected in patients with AML who have relapsed following quizartinib treatment [26, 28]. However, in those assays, inhibition of cell viability for cells expressing FLT3-F691 was 10-fold to 20-fold weaker than for cells expressing FLT3-ITD, suggesting potential efficacy depending on the exposure level achieved in patients. 
Fig. 5 Gilteritinib significantly decreases leukemic burden and increases survival in an intra-bone marrow transplantation model of AML. Female NOD-SCID mice engrafted with MV4-11-luc cells were treated once daily with vehicle (a) or $30 \mathrm{mg} / \mathrm{kg}$ gilteritinib (•) for 56 days beginning on day 15 . Bar represents treatment period. a MV4-11-luc cell bone marrow infiltration was monitored using whole-body imaging. Data presented as mean \pm SEM for $n=10$ mice/group. $* * * P<0.001$ compared with the value of the control group on day 42

(Student's t-test). b

Representative whole-body bioluminescence images from day 21 and day 42 are shown. c Kaplan-Meier analysis curve of mouse survival. $* * * P<0.001$ compared with the value of the control group (Log-rank test) a

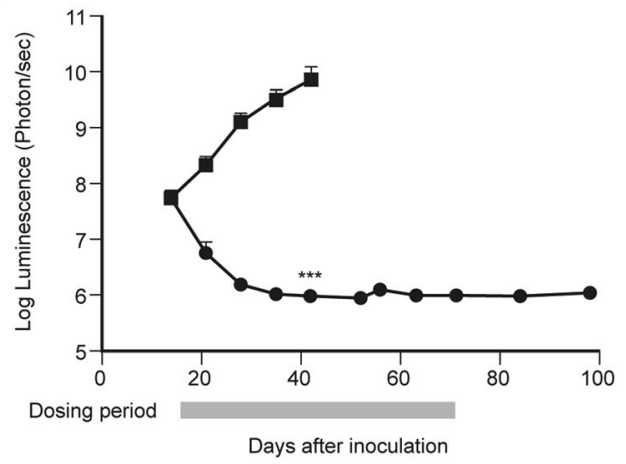

b
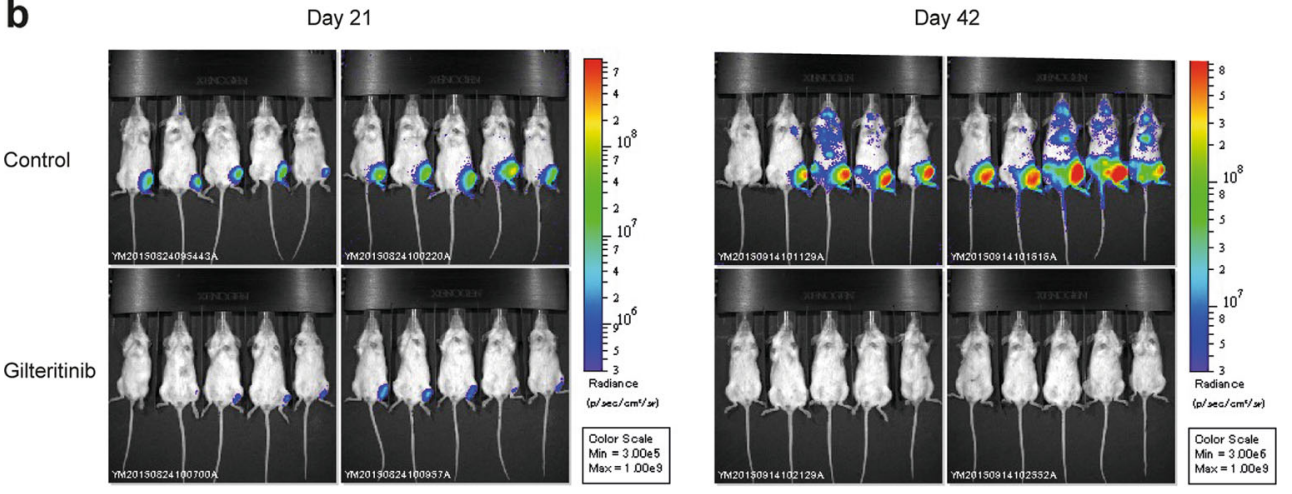

C

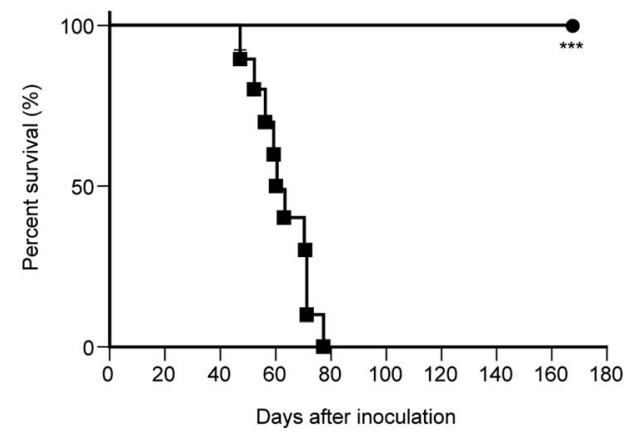

Although several similarities exist between gilteritinib and crenolanib (eg, effective inhibition of FLT3-D835Y and high selectivity for FLT3 compared with c-KIT), crenolanib treatment showed limited efficacy at the maximum tolerated dose in a xenograft mouse model in which MV4-11-luc cells were inoculated by intravenous injection, resulting in a significant survival benefit without a complete reduction in tumor cells [24]. By contrast, $30 \mathrm{mg} / \mathrm{kg}$ gilteritinib induced a significant reduction in bioluminescence from tumor cells to levels near background bioluminescence and improved survival such that all mice in the gilteritinib-treatment group remained alive during the experimental period. Furthermore, $30 \mathrm{mg} / \mathrm{kg}$ gilteritinib treatment continued to suppress the bioluminescence around 3 months post treatment, suggesting that this dose is also effective against minimum residual disease in bone marrow in this model. Overall, the cellular and animal model data suggest that gilteritinib may be advantageous compared with the other FLT3 inhibitors in development because it effectively blocks mutated FLT3 (both FLT3-ITD and FLT3-D835Y).

In addition to the strong preclinical inhibition of FLT3, gilteritinib also targets AXL. In vitro studies have shown that AXL is important for both wild-type and mutant FLT3 activation, suggesting that AXL may have a role in the pathobiology of AML [17]. Furthermore, preclinical evidence in FLT3-ITD expressing cells suggests that activation of AXL may be required for the development of acquired resistance to FLT3 inhibitors [19]. Additional in vivo studies have demonstrated that blocking AXL can suppress the growth of FLT3-ITD AML [17], decrease tumor size [16], and block the activation of cellular survival pathways while upregulating the apoptotic pathway [16]. Thus, the preliminary preclinical data presented herein, showing that gilteritinib inhibits AXL at concentrations 
similar to that for FLT3, suggest that dual inhibition of this pathway may lead to improved efficacy in the treatment of AML; however, it remains unclear whether AXL inhibition played a role in the tumor regression and improved survival induced by gilteritinib in the IBMT model.

Although these preclinical studies clearly demonstrate the potent activity of gilteritinib on FLT3 coupled with minimal activity against c-KIT, a few limitations should be noted. While these data are suggestive of a possible enhanced benefit to blocking both FLT3 and AXL, further studies are needed to fully characterize and understand the effect of gilteritinib on AXL in preclinical models thereby helping to fully elucidate the role of AXL in FLT3-mutated AML. In addition, understanding the effect of gilteritinib in cells from patients with AML may help shed light on the clinical implications of treatment with a FLT3-mutant inhibitor because the patient's cells will be heterogeneous for the FLT3 mutation.

Overall, gilteritinib showed consistently potent inhibition of FLT3 in preclinical studies and demonstrated strong efficacy in FLT3-mutant tumor models. Furthermore, initial in vitro assays suggest that gilteritinib effectively blocks AXL activity while having minimal effect on c-KIT. Based on these data, it can be postulated that gilteritinib may lead to a reduced blast number and prolonged survival in patients with FLT3-ITD- or FLT3D835Y-positive AML. This hypothesis requires confirmation in clinical studies.

Acknowledgements This research was supported by research funding from Astellas Pharma Inc. Financial support for the manuscript development, including writing and editorial assistance by Choice Healthcare Solutions (Chicago, IL), was provided by Astellas Pharma Inc. Gilteritinib was discovered by Astellas Pharma Inc. in collaboration with Kotobuki Pharmaceutical Co., Ltd.

Author's contributions MM contributed to the conceptual ideas, experimental design, data interpretation, and critical writing. NK, YU, MY, RT, and RS designed and performed experiments and analyzed and interpreted the data. IS provided the materials. KM designed the in silico part of the study, performed the calculations, and prepared the manuscript and figs. SK supervised the study.

\section{Compliance with ethical standards}

Animal studies All applicable international, national, and institutional guidelines for the care and use of animals were followed. All procedures performed in studies involving animals were in accordance with the ethical standards of Astellas Pharma, Inc., where the studies were conducted.

Human studies This article does not contain any studies with human participants performed by any of the authors.

Disclosure statement $\mathrm{MY}$ is an employee of Astellas Research Technologies Co., Ltd. RS is a former employee of Astellas Pharma Inc. All other authors are employees of Astellas Pharma Inc.; IS holds a patent on WO2010128659. This study was funded by Astellas Pharma, Inc.

Open Access This article is distributed under the terms of the Creative Commons Attribution 4.0 International License (http:// creativecommons.org/licenses/by/4.0/), which permits unrestricted use, distribution, and reproduction in any medium, provided you give appropriate credit to the original author(s) and the source, provide a link to the Creative Commons license, and indicate if changes were made.

\section{References}

1. Estey E, Dohner H (2006) Acute myeloid leukaemia. Lancet 368(9550):1894-1907

2. Grunwald MR, Levis MJ (2015) FLT3 tyrosine kinase inhibition as a paradigm for targeted drug development in acute myeloid leukemia. Semin Hematol 52(3):193-199

3. Weisberg E, Boulton C, Kelly LM, Manley P, Fabbro D, Meyer T, Gilliland DG, Griffin JD (2002) Inhibition of mutant FLT3 receptors in leukemia cells by the small molecule tyrosine kinase inhibitor PKC412. Cancer Cell 1(5):433-443

4. Kantarjian H, O'Brien S, Cortes J, Giles F, Faderl S, Jabbour E, Garcia-Manero G, Wierda W, Pierce S, Shan J, Estey E (2006) Results of intensive chemotherapy in 998 patients age 65 years or older with acute myeloid leukemia or high-risk myelodysplastic syndrome: predictive prognostic models for outcome. Cancer 106(5):1090-1098

5. Slovak ML, Kopecky KJ, Cassileth PA, Harrington DH, Theil KS, Mohamed A, Paietta E, Willman CL, Head DR, Rowe JM, Forman SJ, Appelbaum FR (2000) Karyotypic analysis predicts outcome of preremission and postremission therapy in adult acute myeloid leukemia: a southwest oncology group/eastern cooperative oncology group study. Blood 96(13):4075-4083

6. Pettit K, Odenike O (2015) Defining and treating older adults with acute myeloid leukemia who are ineligible for intensive therapies. Front Oncol 5:280

7. Dombret H, Gardin C (2016) An update of current treatments for adult acute myeloid leukemia. Blood 127(1):53-61

8. Stirewalt DL, Radich JP (2003) The role of FLT3 in haematopoietic malignancies. Nat Rev Cancer 3(9):650-665

9. Pratz KW, Luger SM (2014) Will FLT3 inhibitors fulfill their promise in acute meyloid leukemia? Curr Opin Hematol 21(2):72-78

10. Nakao M, Yokota S, Iwai T, Kaneko H, Horiike S, Kashima K, Sonoda Y, Fujimoto T, Misawa S (1996) Internal tandem duplication of the flt3 gene found in acute myeloid leukemia. Leukemia 10(12):1911-1918

11. Yamamoto Y, Kiyoi H, Nakano Y, Suzuki R, Kodera Y, Miyawaki S, Asou N, Kuriyama K, Yagasaki F, Shimazaki C, Akiyama H, Saito K, Nishimura M, Motoji T, Shinagawa K, Takeshita A, Saito H, Ueda R, Ohno R, Naoe T (2001) Activating mutation of D835 within the activation loop of FLT3 in human hematologic malignancies. Blood 97(8):2434-2439

12. Frohling S, Schlenk RF, Breitruck J, Benner A, Kreitmeier S, Tobis K, Döhner H, Döhner K, AML Study Group Ulm. Acute myeloid leukemia (2002) Prognostic significance of activating FLT3 mutations in younger adults (16 to 60 years) with acute myeloid leukemia and normal cytogenetics: a study of the AML study group Ulm. Blood 100(13):4372-4380

13. Abu-Duhier FM, Goodeve AC, Wilson GA, Care RS, Peake IR, Reilly JT (2001) Identification of novel FLT-3 Asp835 mutations in adult acute myeloid leukaemia. Br J Haematol 113(4):983-988

14. Bacher U, Haferlach C, Kern W, Haferlach T, Schnittger S (2008) Prognostic relevance of FLT3-TKD mutations in AML: the combination matters-an analysis of 3082 patients. Blood 111(5):2527-2537

15. Whitman SP, Ruppert AS, Radmacher MD, Mrózek K, Paschka P, Langer C, Baldus CD, Wen J, Racke F, Powell BL, Kolitz JE, Larson RA, Caligiuri MA, Marcucci G, Bloomfield CD (2008) FLT3 D835/I836 mutations are associated with poor disease-free survival 
and a distinct gene-expression signature among younger adults with de novo cytogenetically normal acute myeloid leukemia lacking FLT3 internal tandem duplications. Blood 111(3):1552-1559

16. Ben-Batalla I, Schultze A, Wroblewski M et al (2013) Axl, a prognostic and therapeutic target in acute myeloid leukemia mediates paracrine crosstalk of leukemia cells with bone marrow stroma. Blood 122(14):2443-2452

17. Park IK, Mishra A, Chandler J, Whitman SP, Marcucci G, Caligiuri MA (2013) Inhibition of the receptor tyrosine kinase Axl impedes activation of the FLT3 internal tandem duplication in human acute myeloid leukemia: implications for Axl as a potential therapeutic target. Blood 121(11):2064-2073

18. Janning M, Ben-Batalla I, Loges S (2015) Axl inhibition: a potential road to a novel acute myeloid leukemia therapy? Expert Rev Hematol 8(2):135-138

19. Park IK, Mundy-Bosse B, Whitman SP, Zhang X, Warner SL, Bearss DJ, Blum W, Marcucci G, Caligiuri MA (2015) Receptor tyrosine kinase $\mathrm{Axl}$ is required for resistance of leukemic cells to FLT3-targeted therapy in acute myeloid leukemia. Leukemia 29(12):2382-2389

20. Lee MW, Kim HJ, Yoo KH, Kim DS, Yang JM, Kim HR, Noh YH, Baek H, Kwon H, Son MH, Lee SH, Cheuh HW, Jung HL, Sung KW, Koo HH (2012) Establishment of a bioluminescent imagingbased in vivo leukemia model by intra-bone marrow injection. Int $\mathrm{J}$ Oncol 41(6):2047-2056

21. Konig H, Levis M (2015) Targeting FLT3 to treat leukemia. Expert Opin Ther Targets 19(1):37-54
22. Mackarehtschian K, Hardin JD, Moore KA, Boast S, Goff SP, Lemischka IR (1995) Targeted disruption of the flk2/flt3 gene leads to deficiencies in primitive hematopoietic progenitors. Immunity 3(1):147-161

23. Galanis A, Levis M (2015) Inhibition of c-kit by tyrosine kinase inhibitors. Haematologica 100(3):e77-e79

24. Zimmerman EI, Turner DC, Buaboonnam J, Hu S, Orwick S, Roberts MS, Janke LJ, Ramachandran A, Stewart CF, Inaba H, Baker SD (2013) Crenolanib is active against models of drugresistant FLT3-ITD-positive acute myeloid leukemia. Blood 122(22):3607-3615

25. Zarrinkar PP, Gunawardane RN, Cramer MD, Gardner MF, Brigham D, Belli B, Karaman MW, Pratz KW, Pallares G, Chao Q, Sprankle KG, Patel HK, Levis M, Armstrong RC, James J, Bhagwat SS (2009) AC220 is a uniquely potent and selective inhibitor of FLT3 for the treatment of acute myeloid leukemia (AML). Blood 114(14):2984-2992

26. Smith CC, Wang Q, Chin CS, Salerno S, Damon LE, Levis MJ, Perl AE, Travers KJ, Wang S, Hunt JP, Zarrinkar PP, Schadt EE, Kasarskis A, Kuriyan J, Shah NP (2012) Validation of ITD mutations in FLT3 as a therapeutic target in human acute myeloid leukaemia. Nature 485(7397):260-263

27. Smith CC, Lin K, Stecula A, Sali A, Shah NP (2015) FLT3 D835 mutations confer differential resistance to type II FLT3 inhibitors. Leukemia 29(12):2390-2392

28. Smith CC, Zhang C, Lin KC et al (2015) Characterizing and overriding the structural mechanism of the quizartinib-resistant FLT3 "gatekeeper" F691L mutation with PLX3397. Cancer Discov 5(6):668-679 